\title{
Evaluation of Sentinel-3 SRAL SAR altimetry over Chinese rivers
}

\author{
Jiang, Liguang; Nielsen, Karina; Dinardo, Salvatore; Andersen, Ole Baltazar; Bauer-Gottwein, Peter
}

\section{Published in:}

Remote Sensing of Environment

Link to article, DOI:

10.1016/j.rse.2019.111546

Publication date:

2020

Document Version

Peer reviewed version

Link back to DTU Orbit

Citation (APA):

Jiang, L., Nielsen, K., Dinardo, S., Andersen, O. B., \& Bauer-Gottwein, P. (2020). Evaluation of Sentinel-3 SRAL SAR altimetry over Chinese rivers. Remote Sensing of Environment, 237, [111546].

https://doi.org/10.1016/j.rse.2019.111546

\section{General rights}

Copyright and moral rights for the publications made accessible in the public portal are retained by the authors and/or other copyright owners and it is a condition of accessing publications that users recognise and abide by the legal requirements associated with these rights.

- Users may download and print one copy of any publication from the public portal for the purpose of private study or research.

- You may not further distribute the material or use it for any profit-making activity or commercial gain

- You may freely distribute the URL identifying the publication in the public portal 


\section{Evaluation of Sentinel-3 SRAL SAR Altimetry over Chinese Rivers}

Liguang Jiang ${ }^{\mathrm{a}}$

Karina Nielsen ${ }^{b}$

Salvatore Dinardo ${ }^{\mathrm{c}}$

Ole B. Andersen ${ }^{b}$

Peter Bauer-Gottwein ${ }^{\text {a }}$

${ }^{a}$ Department of Environmental Engineering, Technical University of Denmark, $2800 \mathrm{Kgs}$. Lyngby, Denmark

b DTU Space, National Space Institute, Technical University of Denmark, 2800 kgs. Lyngby, Denmark

${ }^{c}$ He Space, Robert Bosch Strasse 7, 64293 Darmstadt, Germany

* Corresponding author. Email: 1jia@env.dtu.dk 


\section{Abstract}

2 Satellite radar altimetry observations of water surface elevation (WSE) have become an important data source to supplement river gauge records. Sentinel-3 is the first radar altimetry mission operating with a synthetic aperture radar (SAR) altimeter at global scale and with a new on-board tracking system (i.e. openloop), which has great potential in terms of delivering reliable observations of inland water bodies for the next two decades (several future missions include an open-loop tracking mode). In this context, it is very important to investigate the data quality at an early stage. In this study, a comprehensive evaluation of Sentinel-3A (S3A) is conducted at 50 virtual stations (VS) located on a wide range of rivers in China.

The evaluation of Level 1 data shows that, over mountain rivers, a good prior surface elevation estimate onboard is vital to deliver useful datasets using the S3A open-loop tracking system. The Open-Loop Tracking Command version 5 (OLTC V5) has significantly improved the placement of the range window, which was misplaced and resulted in lack of data over many mountain rivers prior to OLTC V5 (March 2019). However, application of S3A over mountain rivers still require careful evaluation, especially before March 2019.

Four retrackers are evaluated including a physical SAR Altimetry Mode Studies and Applications retracker (SAMOSA+), a traditional Offset Center Of Gravity (OCOG), a Primary Peak Center Of Gravity (PPCOG), and a modified Multiple Waveform Persistent Peak (MWaPP+) retracker. For 26 VSs in plain areas, retracked WSE data achieved a root mean square error (RMSE) ranging from $0.12 \mathrm{~m}$ to $0.9 \mathrm{~m}$. The comparison of retracking methods reveals that SAMOSA+, OCOG, and PPCOG are unable to handle multipeak waveforms. But the MWaPP+ can significantly improve the accuracy of the estimated WSE over large rivers, especially when the waveforms are contaminated.

Moreover, our result shows no considerable difference between medium (ca. $300 \mathrm{~m}$ wide) and large (wider than $500 \mathrm{~m}$ ) rivers. Instead, surrounding topography and homogeneity of surroundings are very important factors influencing the shape of a waveform. For rivers surrounded by lakes, man-made channels etc., special care must be taken when processing altimetry data. Dedicated retracking methods, such as $\mathrm{MWaPP}+$, and sophisticated methods for outlier detection are needed to improve precision over such rivers, as demonstrated here for the Yangtze River.

Keywords: Water surface elevation, Radar altimetry, Retracking, Sentinel-3, SAR, Open-loop, OLTC

\section{Introduction}

In many rivers, flow regimes and water volumes are changing due to climate change and intensive human activities (Arnell and Gosling, 2013; Krasovskaia and Gottschalk, 2002). Therefore, it is important to monitor the state of rivers. A fundamental quantity of river hydrology/hydraulics is the stage. The stage of a river/stream is the elevation of the water surface (WSE) above a datum. Stage can be determined either by manual observation from staff gauges or through automated sensors, such as pressure transducers, optical 
sensors, radio detection and ranging sensors (Sauer and Turnipseed, 2010). However, the density of traditional ground-based monitoring stations is very limited and there exist institutional barriers that limit data sharing. Lack of data constrains our ability to observe and predict hydrological events, e.g. flooding, especially in mountain areas. Several large rivers, originating in and flowing through the Himalayan region, are not well monitored. However, these rivers are important for the one sixth of the global human population that live in these river basins (Immerzeel et al., 2010). Hence, there is an urgent need for alternative and efficient techniques for stage monitoring for these poorly gauged or even ungauged river systems.

In recent decades, satellite radar altimetry has been successfully used to deliver observations of WSE. Numerous studies have used altimetry observations to extract WSE for inland water bodies such as lakes and rivers (Jiang et al., 2017a; Kleinherenbrink et al., 2015; Nielsen et al., 2015; Villadsen et al., 2016 among others). Moreover, altimetry-derived WSE has been used for hydrologic/hydrodynamic modelling (Domeneghetti et al., 2014; Jiang et al., 2019b; Kittel et al., 2018; Liu et al., 2015; Michailovsky et al., 2013; Schneider et al., 2018a, 2018b) and river discharge estimation (Leon et al., 2006; Michailovsky et al., 2012; Sichangi et al., 2016; Tarpanelli et al., 2013). A recent review paper (Jiang et al., 2017b) has summarized the hydrological applications of satellite altimetry with a specific focus on CryoSat-2, which is the first satellite altimetry mission with Synthetic Aperture Radar (SAR) altimeter and has demonstrated the value of SAR altimetry (Nielsen et al., 2017; Villadsen et al., 2016). Thanks to the finer along-track resolution, SAR altimetry is less influenced by land contamination, islands, etc. compared to conventional low resolution mode (LRM) altimetry, especially in coastal areas (Dinardo et al., 2018; Idžanović et al., 2018). The accuracy of satellite altimetry derived WSE has been improved for last 25 years leading to accuracy on the order of decimeters over rivers (Berry et al., 2005; Normandin et al., 2018) due to a number of factors, e.g. smaller footprint, the advancement of orbit determination, and retracking, etc. (Wingham et al., 2006).

The Sentinel-3 mission is part of the European Commission's Copernicus programme. Sentinel-3 is designed as a constellation of two identical polar orbiting satellites, i.e. Sentinel-3A (S3A) and Sentinel-3B (S3B) for the provision of high-resolution operational marine and land monitoring services. S3A and S3B were launched in February 2016 and April 2018, respectively. The dual-frequency (Ku- and C-band) Synthetic Aperture Radar Altimeter (SRAL) on board the Sentinel-3 mission, which is based on the heritage from the CryoSat-2 and the Jason missions, has some new features. It is the first satellite altimeter to provide global coverage in SAR mode, which provides observations at a finer along-track resolution. Another important characteristic is the on-board tracking mode, i.e. open-loop tracking mode, which controls the return echo acquisition phase by properly setting its range window in time, based on a prior surface elevation information on-board. A recent study shows that the data quality of Sentinel-3 is quite good over the Namco Lake (Jiang et al., 2019a). Compared to CryoSat-2 and SARAL/AltiKa, Sentinel-3 is less affected by topography in lake coastal regions (Jiang et al., 2019a). However, virtually no information is available on its performance especially over regions with significant elevation variations. One would expect that the open- 
loop tracking mode offers enhanced capabilities with respect to the closed-loop tracking mode over this type of terrain, but this remains to be demonstrated with real data and results.

With these new characteristics, Sentinel-3 is expected to deliver reliable observations of WSE for inland water bodies. However, the performance of Sentinel-3 open-loop mode over rivers is not widely reported and many questions remain to be answered. (1) Can Sentinel-3 deliver reliable measurements of WSE for mountain rivers, especially those flowing through steep topographic relief, e.g. upstream of the Yangtze, Yellow rivers, etc.? (2) What is the accuracy of Sentinel-3 derived WSE? (3) Can Sentinel-3 observe medium rivers (ca. $300 \mathrm{~m}$ wide)? To answer these questions, we exhaustively evaluate the Sentinel-3A data during the past three years over Chinese rivers with varying width and topography.

\section{Background}

In radar altimetry, a microwave pulse is transmitted by the altimeter, then reflected by the surface, and finally part of its echo is recorded at the altimeter (Fig. 1). Therefore, to record the echoes from a river, the range window (typically $60 \mathrm{~m}$ ) must be positioned correctly in time (i.e. window delay). This is done by the on-board tracking system in e.g. closed-loop or open-loop (Rosmorduc et al., 2011). The closed-loop mode has two operational phases: first, the acquisition phase is to detect useful signal and initialize the tracker's range window; second, the tracking phase aims at maintaining the range window in the correct position, i.e. the leading-edge point of the echo is placed within the range window (typically at one third of the full window) (Chelton et al., 2001). However, since the closed-loop tracking system relies on previous measurements to position the current range window, previous radar altimetry missions, such as Envisat, Jason-1/-2, CryoSat2, etc., have had difficulties to measure WSE of rivers which are surrounded by or adjacent to steep mountains. In other words, the echoes reflected by rivers are not (correctly) recorded due to the position of the range window as shown in Fig. 1b. Dehecq et al. (2013) showed the inability of CryoSat-2 to track the regions of interest over the Himalayan range due to the closed-loop tracking. Biancamaria et al. (2018) reported this issue over French rivers. Similar issues occur in the upper part of the Yangtze and Yellow rivers in mountain areas (Jiang et al., 2017a). 
(a)

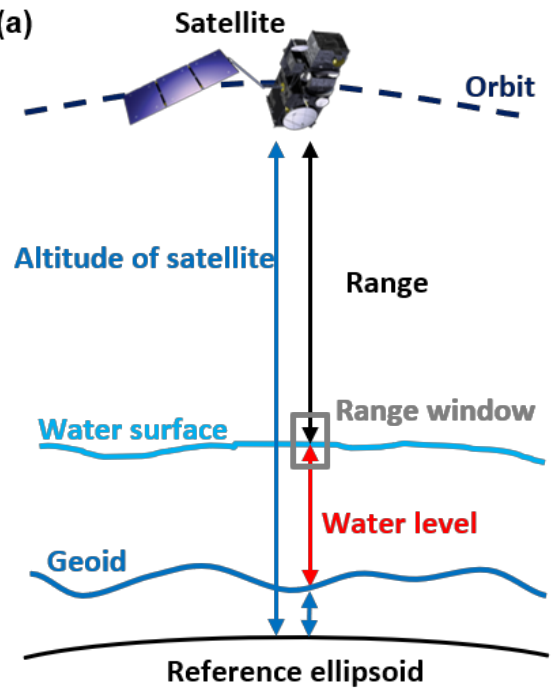

(b)
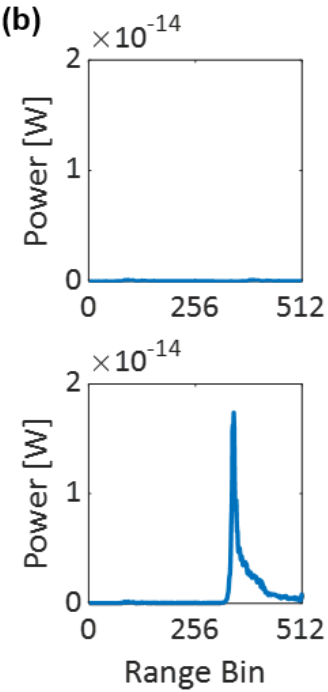
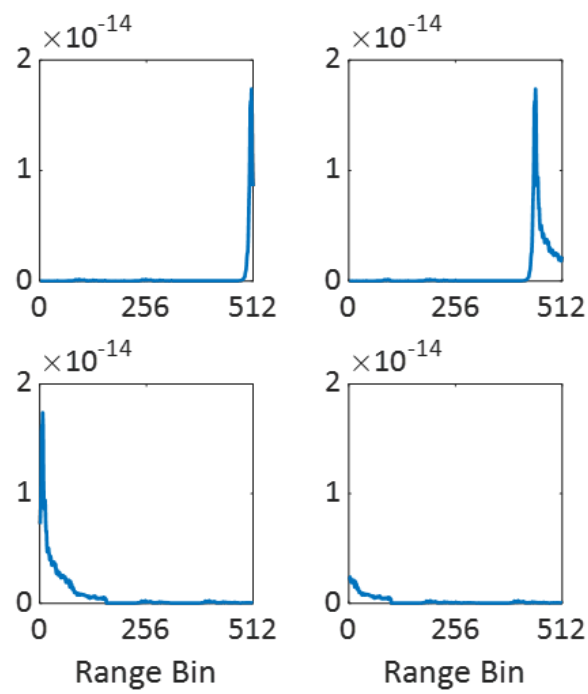

Fig. 1. Illustration of the principle of satellite altimetry (a) and waveforms (b). In (b), six scenarios of range window positioned differently, demonstrating the corresponding recorded echoes. In the first two cases, range windows are positioned too early in time so the waveforms only record background noise or small portion of the leading edge; the next two are correctly positioned and the peak is fully recorded, but the fourth is better comparatively; in the last two cases, range windows are positioned too late to record the full peak.

The open-loop mode operates based on prior along-track surface elevations (Digital Elevation Model (DEM)) uploaded on-board by which the altimeter range window can be properly positioned. Therefore, this mode does not require an acquisition phase and there is no tracking loss anymore (Desjonquères et al., 2010). The study of Biancamaria et al. (2018) shows the benefit of open-loop tracking implemented on Jason-3 over French rivers. However, the quality of the on-board DEM is directly linked with the tracker performance.

Considering the relatively small size of the range window (e.g. $60 \mathrm{~m}$ for Sentinel-3), the prior elevation information on-board is the key factor to deliver reliable echoes in mountain areas. Given that annual amplitudes of river water level fluctuations are generally smaller than $15 \mathrm{~m}, \mathrm{a} \pm 5 \mathrm{~m}$ accuracy can ensure the range window enclosing the elevation of a river. However, due to memory limitations, the Sentinel-3 onboard DEM is actually a pseudo-DEM controlled through the Open-Loop Tracking Command (OLTC) table, which is generated from the 30" ACE2 DEM by applying a surface mask, i.e. Globcover (300 m resolution) for OLTC V4 on S3A (Sophie Le Gac, personal communication, 2019). More specifically, at locations that are defined as water, the ACE2 DEM elevation value is assigned to the range window based on predefined OLTC tables. At other locations, the pseudo-DEM values are interpolated along track using available values located closest to the present position along the same track (Fig. 2). Thus, the accuracy of the water surface mask is another important factor determining data availability (Desjonquères et al., 2010). For example, as illustrated in Fig.2, if small river number 4 is not identified as water, no a-priori elevation is assigned to the 

3.

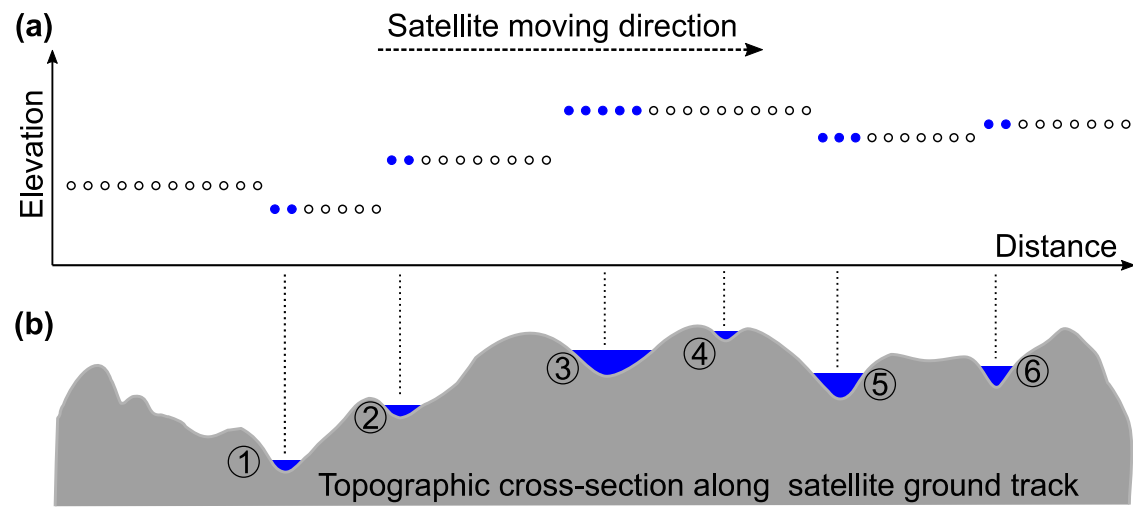

Fig. 2. Illustration of on-board pseudo-DEM available in the OLTC. (a) A-priori elevation stored in OLTC. Blue dots indicate that a-priori elevation is available while circles indicate no a-priori elevation available.

When no a-priori information is provided, the on-board tracking function uses the latest available elevation. (b) Topographic cross-section along a satellite ground track. Rivers are in blue.

It should be noted that S3A has been working in open-loop mode with the OLTC V4 until $1^{\text {st }}$ March 2019. However, only 4 virtual stations (VS) are included in OLTC V4 across China, which means the range window was positioned based on interpolated incorrect values for most VSs. During 2019-03-01 and 201903-09, the instrument was working in closed-loop due to the update of OLTC from V4 to V5. And since then, S3A is working in open-loop again with a new OLTC consisting of around 33000 VSs and covering the whole area between $60^{\circ} \mathrm{N}$ to $60^{\circ} \mathrm{S}$ (Blumstein et al., 2019). Details about the distribution of VSs defined in OLTC V5 are available on the "OLTC for Hydrology" platform https://www.altimetry-hydro.eu/.

\section{Materials and methods}

\subsection{Study region}

China has a vast network of rivers across different landscapes and topographic regions, which provide a good test bed to validate Sentinel-3 SAR altimetry data over rivers. Most parts of China were covered by the open-loop tracking mode except the most southern part, i.e. the Pearl River basin before $1^{\text {st }}$ March 2019 under OLTC V4. Since then, the whole study domain is covered by open-loop under OLTC V5. In this study, 50 VSs are selected based on their distance to gauge stations as well as the width and altitude of rivers. As shown in Fig. 3, these VSs are located across China. Those located in northeastern and eastern parts are characterized by low elevation and relatively flat topography. These sites allow us to evaluate the performance of SAR altimetry over diverse river widths. Moreover, to assess the performance of open-loop tracking over steep topography, some VSs located in the mountain areas are included. Validation of VSs in 

areas where satellite altimetry has the highest value.

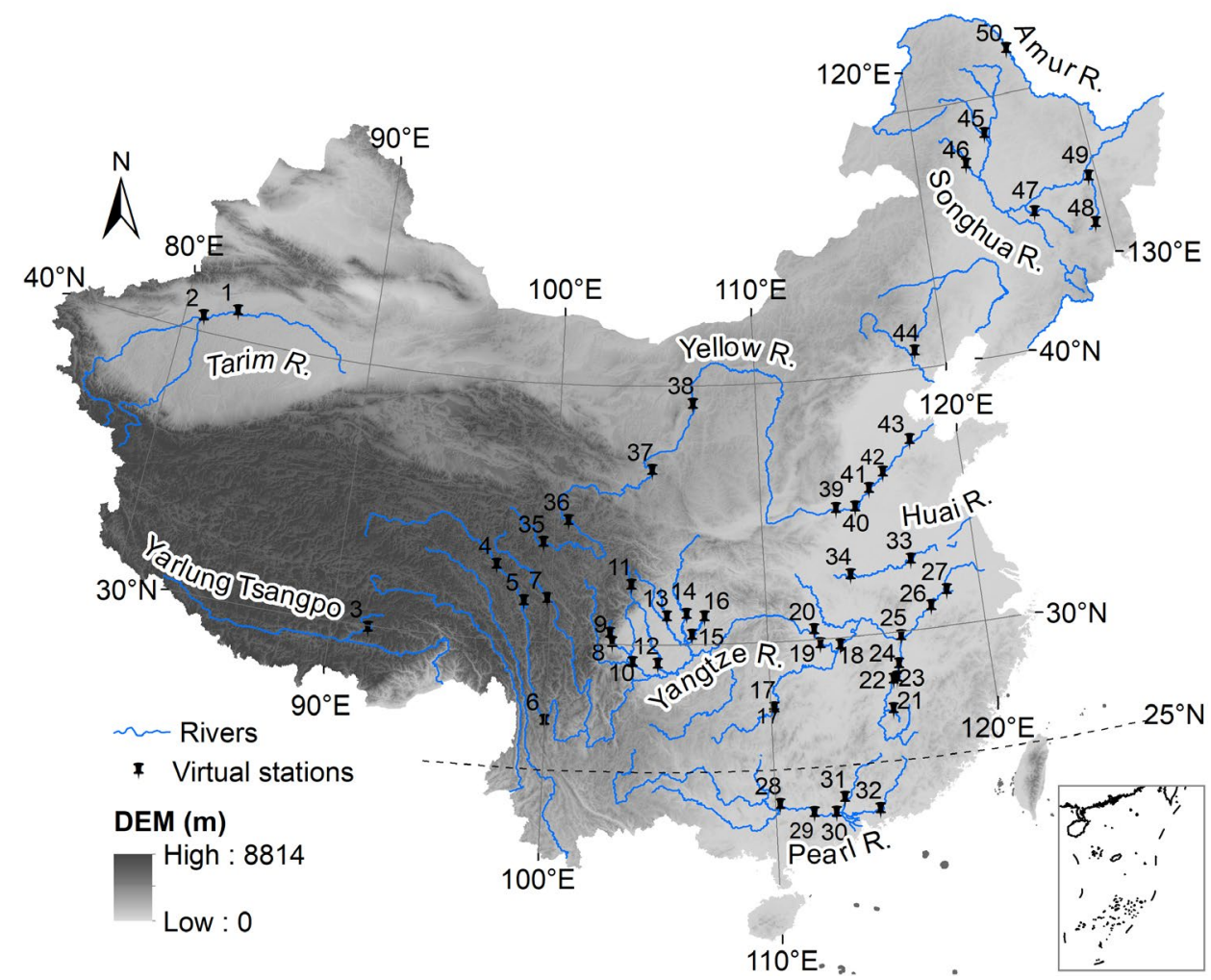

Fig. 3. Study domain showing the rivers and Sentinel-3A virtual stations (VS) investigated in this study. VSs with latitude higher than $25^{\circ}$ are covered by open-loop control mode and those with latitude lower than $25^{\circ}$ are in closed-loop under OLTC V4. The study domain is fully covered by open-loop under OLTC V5.

\subsection{Sentinel-3 L1b and L2 data processing}

We collect Level-1b and Level-1b stack data (July 2016 to June 2019) from ESA GPOD (Grid Processing On Demand) SARvatore service available at https://gpod.eo.esa.int/services/SENTINEL3 SAR/ (Dinardo et al., 2014). GPOD allows users to tailor configuration for the data processing. We obtain the Level-1b and Level-1b stack data according to the following steps (Dinardo et al., 2018; Dinardo and Benveniste, 2013). First, a Hamming weighting window is applied on the burst data to mitigate the effect of side-lobes ambiguities or the impact of "ghost" signals originating from very high scattering targets that are placed outside the main-lobe of the synthetic beam. Second, beam-forming and beam-steering are applied to form the fan of synthetic Doppler beams and to make the Doppler footprints co-located with the surface sample locations, respectively. Third, FFT zero-padding (over-sampling the return radar waveform by factor of two) is applied to sample very peaky echoes from bright targets. Finally, double extension of the receiving window (temporal use to store stacks) is applied to mitigate on-board tracker errors on rough topography (Dinardo et al., 2018). 
Based on Level 1b stack and Level 1b waveform, we calculate the following parameters. Note that, some statistics of the stack are derived from the Gaussian fit of the range integrated power (RIP). Fig. 4 shows an example of these metrics.

- $\quad$ Stack Peakiness (SP): maximum fitted RIP divided by the sum of fitted RIP; High SP indicates river-like (quasi-specular) surfaces

- Maximum power (MP): maximum value of a waveform

- $\quad$ Pulse Peakiness (PP): maximum value of a waveform divided by the sum of the waveform; High PP indicates a more specular reflection.

- Number of peaks (NP): the number of peaks (larger than 25\% of MP) in a waveform (threshold of $25 \%$ is based on Villadsen et al., 2016); High NP indicates multiple bright targets.

(a) Stack

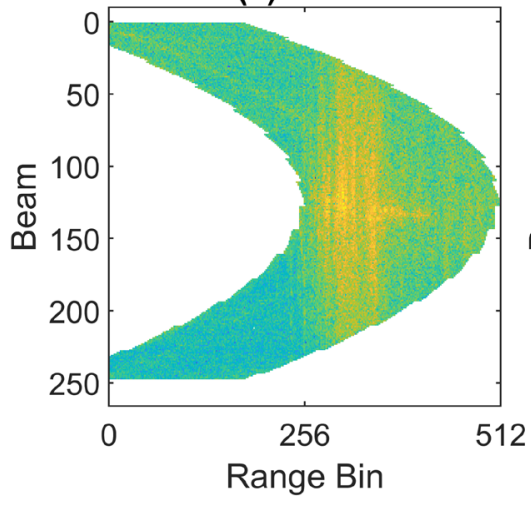

(b) RIP

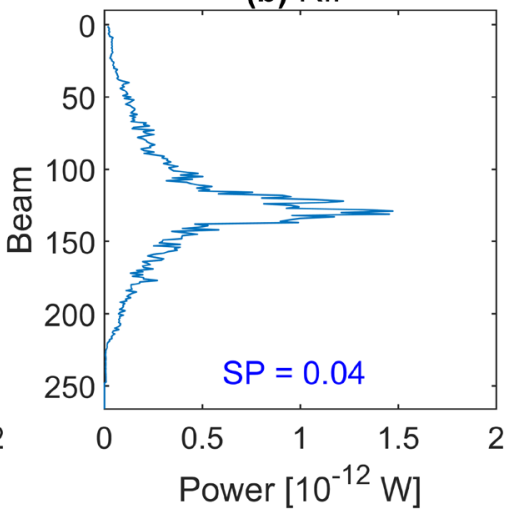

(c) Waveform

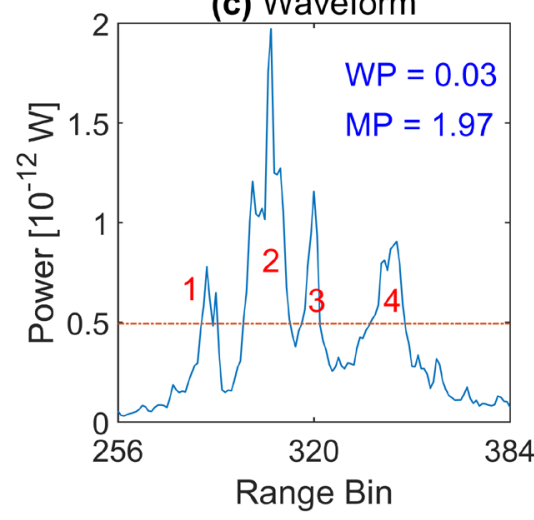

Fig. 4. One exemplary L1b stack and corresponding RIP and waveform at VS 18. (b) RIP is calculated by summing all stacks at each beam, i.e. along $\mathrm{x}$-axis of (a); In this case, stack peakiness is 0.04 , indicating a very narrow and peaky RIP. (b) Waveform is obtained from the bin-wise summation of stacks, i.e. along yaxis of (a); Only echoes between 256 and 384 are shown for clarity. Based on 25\% of MP, four peaks are identified as indicated by numbers in (c).

To retrieve accurate range, $\mathrm{L} 1 \mathrm{~b}$ needs to be further processed by applying retracking algorithms. In this study, we use GPOD default SAMOSA+ (Dinardo et al., 2018) retracker for GPOD data. To compare SAMOSA + with other widely used retrackers, we also implement OCOG and primary peak COG (PPCOG) on L1b waveforms (Jain et al., 2015). The WSE, i.e. L2 data, is the $20 \mathrm{~Hz}$ altimetric height above the reference ellipsoid corrected for all corrections, including instrumental errors, range correction, and geophysical corrections (dry and wet troposphere, ionosphere, solid earth tide, polar tide).

In addition, for rivers wider than $500 \mathrm{~m}$, we also implemented a modified multiple waveform persistent peak (MWaPP+) retracker (Villadsen et al., 2016). The assumption is that one peak reflected by the river occurs in all waveforms, but other peaks reflected by other bright targets or off-nadir water bodies are less likely to be persistent. In this study, our approach is slightly different from the MWaPP+ proposed by Villadsen et al. (2016). For each track, a new waveform is formed by taking the median of all available 
waveforms. Median value is robust because it is less sensitive to extreme values. Instead of the first subwaveform, we extract the sub-waveform with largest peak assuming that the persistent peak dominates the full waveform. Then the $80 \%$ threshold of the amplitude of the sub-waveform is used to locate the retracking point.

\subsection{WSE data editing}

To determine which radar measurements are reflected by water surfaces, we use a water surface mask (Pekel et al., 2016) to select L2 observations. Specifically, we use the occurrence dataset with a threshold of 10, i.e. water occurrence frequency is $10 \%$ between 1984 and 2015. In addition, we also use DEM $\pm 10 \mathrm{~m}$ to discard outliers. Therefore, for some passes, there may be no observation for narrow rivers. The median value of all retracked WSE of the same pass for a given VS is used to construct altimetric WSE time series. It should be noted that, regarding MWaPP+ retracked WSE, there is only one value for each pass. Obvious outliers in the dry season are not counted when calculating the RMSE.

\subsection{Gauging station data}

The in-situ network consists of 50 hydrological gauge stations, which are within $3 \mathrm{~km}$ distance to the corresponding VS. In-situ daily stage data are collected for validation of Sentinel-3A WSE data during January 2016 - June 2019 from the Hydroinfo website managed by the Ministry of Water Resources of China (http://xxfb.mwr.cn/index.html). Both stage data and S3A WSE are transformed to water level anomaly by subtracting the mean value for comparison purposes due to unknown local vertical datums.

\section{Results and discussion}

Given that $\mathrm{L} 2$ is retrieved from L1 data, in this section, we start with the investigation of L1b data with the aim of understanding whether S3A works in open-loop mode with different OLTCs, especially in mountain areas. Subsequently, we present the L2 WSE retrieval and validation.

\subsection{Characteristics of L1b data (stacks and waveforms) under OLTC V4 and OLTC V5}

Fig. 5 shows the four statistical metrics under OLTC V4 and OLTC V5 for the two periods, i.e. before $1^{\text {st }}$ March and after $9^{\text {th }}$ March 2019. PP and SP agree well with each other and are larger than 0.1 and 0.2 for most VSs, respectively. This is to say the waveform is peaky and stack is narrow, indicating that the echoes are less sensitive to land contaminations. Rivers have smooth mirror-like surfaces which usually exhibit quasi-specular reflection instead of diffusive reflection. Therefore, these metrics can be used to filter out some abnormal waveforms. Besides, the metric of NP is a more robust measure to identify whether the waveform is contaminated by multiple bright objects. It is well known that multi-peak waveforms contain echoes from different ground surfaces, and it is difficult to identify the one reflected by the nadir river surface. Taking VSs 12, 13, 16 and 17 as examples, although their PP and SP are large (see Figs. 5a and b), their NP 
are generally larger than 1. These multi-peak waveforms result in high RMSE of estimated WSE (shown in following section). It is well known that echoes reflected by a calm water surface have a higher power (c.a. $10^{-16}-10^{-12} \mathrm{~W}$ ). Lower power (Fig.4d) indicates the echoes are probably not reflected by water, and therefore some VSs have very small PP, SP, and large NP (Fig.5). Waveforms of these VSs only record noisy echoes that are a few orders of magnitude smaller than those reflected by water surface. Therefore, the maximum power should first be checked when processing L2 data from rivers. It is worth noting that the four metrics are similar in the two periods for most VSs, but hugely different in the two periods for 11 VSs, especially the maximum power (Fig. 5d).

(a) Pulse Peakiness

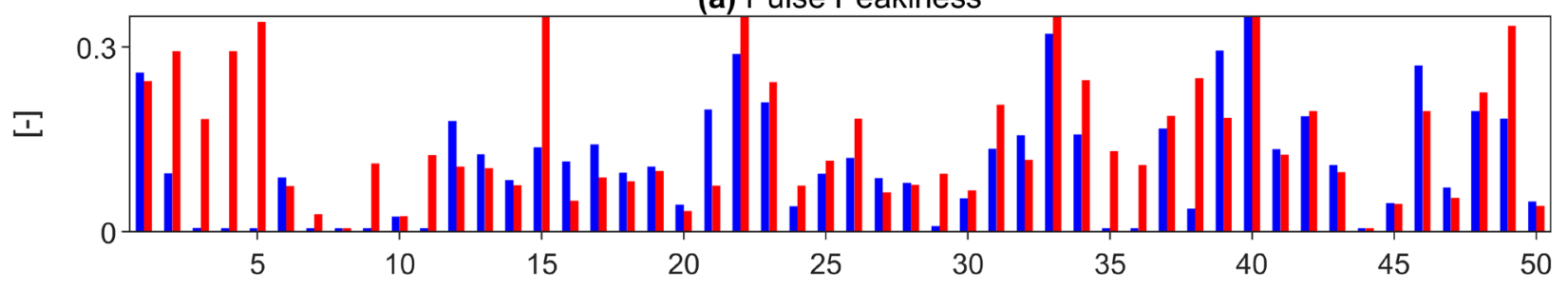

(b) Stack Peakiness

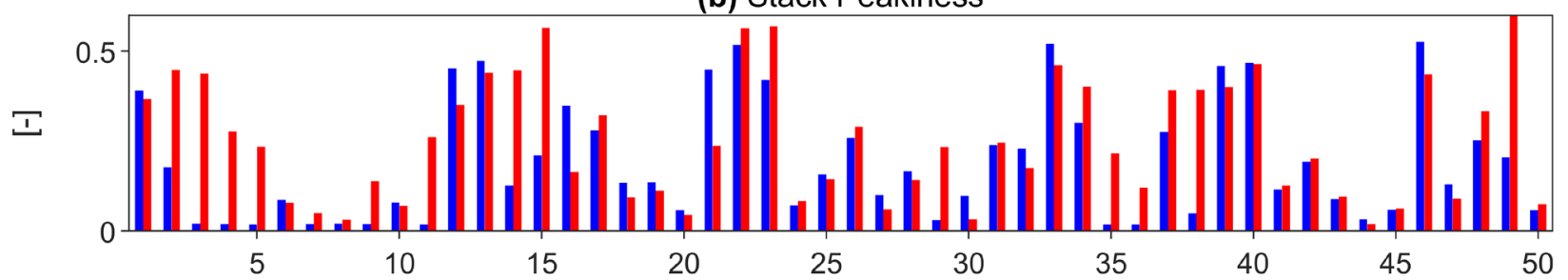

(c) Number of Peaks

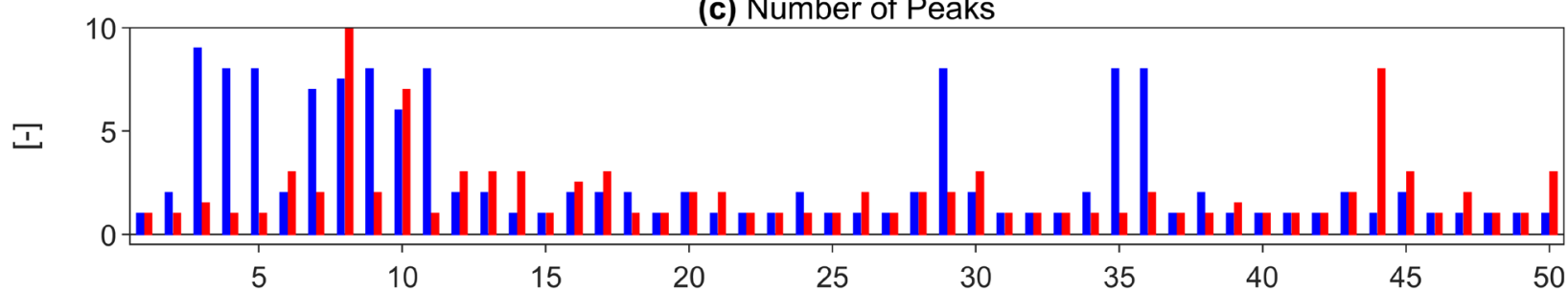

(d) Maximum Power

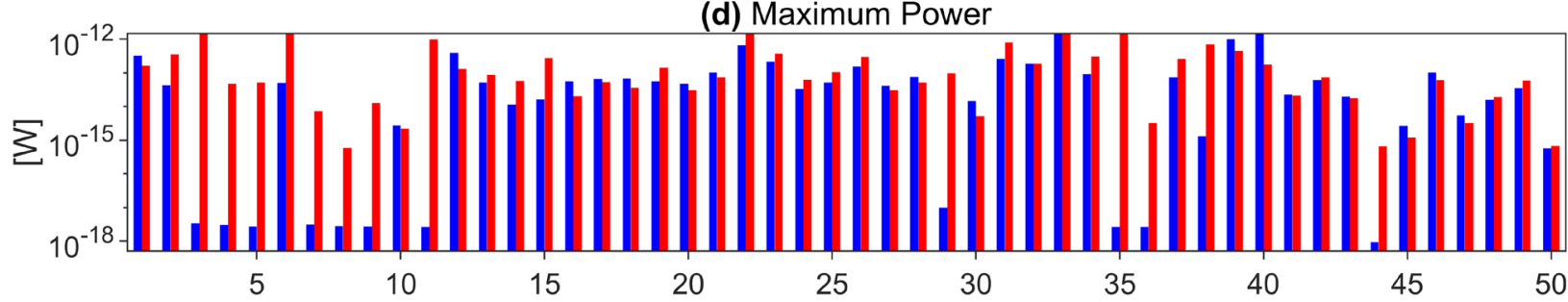

Fig. 5. Characteristics of Sentinel-3A stack and waveform for $50 \mathrm{VSs}$ (x-axis). Blue bars stand for metrics before $1^{\text {st }}$ March 2019 (OLTC V4) while red bars are metrics after $9^{\text {th }}$ March 2019 (OLTC V5). Numbers along $\mathrm{x}$-axis are IDs of VSs as indicated in Fig. 3. areas? 
As shown in Fig. 5, S3A failed to deliver useful data for 11 VSs, which are mainly located in mountainous areas (Fig. 3) in the period prior to March 2019. The main reason is that the on-board DEM, i.e. OLTC V4 on S3A did not hold a correct terrain height value for these targets, which leads to an incorrectly positioned range window. In other words, the returned waveforms do not include any echoes from the water surface, but they resemble background noise (low power as shown in Fig. 5d). This can be verified by comparing altimetry retrieved WSE with a DEM. For example, in Fig. 6, the S3A-derived height is far from the true water surface elevation of the rivers. Clearly, the range window was completely wrongly positioned. As shown in Fig. 6, the return echoes are in the order of $10^{-18} \mathrm{~W}$. Compared to those reflected by water surfaces, these echoes are very small, which shows that the recorded signals are only background noises (Figs. 6c and d). There is a small difference between these two cases. For track 67, the range window is positioned too high, and thus the signal received is just thermal noise. However, for track 4 , the range window is positioned too low, which means that it is too late to capture the signal returned from the river. Both cases result in very noisy waveforms. This is also revealed by the low values of PP and SP reported in the previous section (VS 36).

This problem can be attributed to the errors in on-board DEM, which arise from the inappropriate interpolation method as implemented on-board to reduce the size of the DEM. As confirmed with OLTC platform website (https://www.altimetry-hydro.eu/), there is no a-priori surface elevation information for all the 11 VSs where S3A failed to detect rivers with the OLTC V4. This also explains the findings of no useful data and degraded data over the Brahmaputra river in a recent study (Huang et al., 2019).

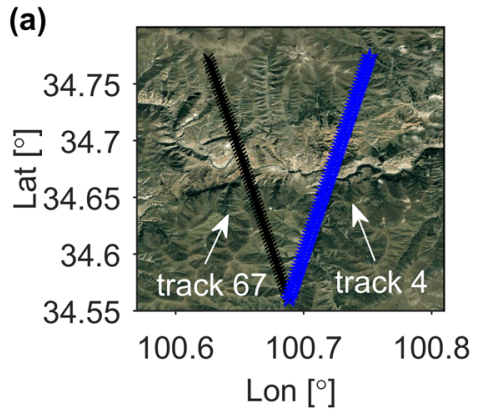

(c)

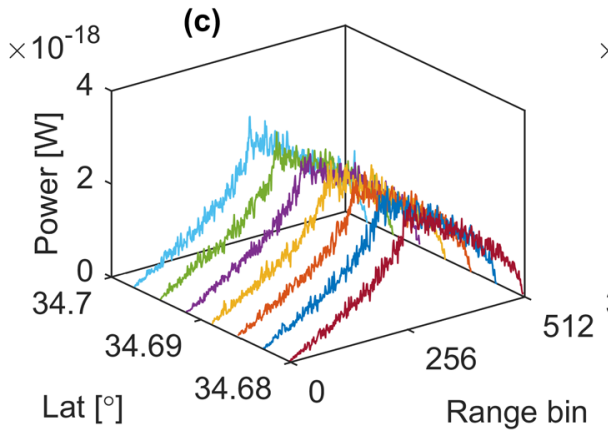

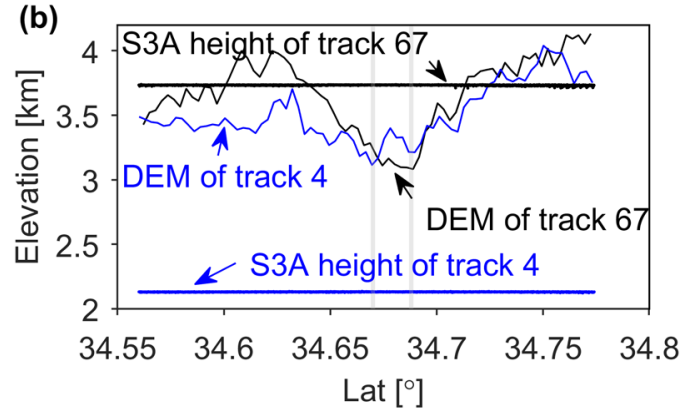

(d)

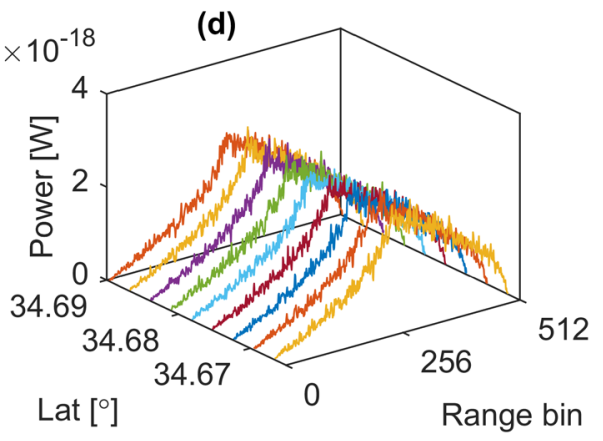




\subsubsection{How does the open-loop mode work with the OLTC V5 since march 2019?}

261

262

263

264

265

266

267

268

269

270

271

272

273

To better explore the detection capability of the open-loop mode, we investigated S3A data obtained with new OLTC V5 since $9^{\text {th }}$ March 2019. A rule of thumb is to check the maximum power of the waveforms. As shown in Fig. 5d, S3A performs better with OLTC V5 in terms of maximum power. Fig. 7 exemplifies the improvement of data under OLTC V5. Clearly, S3A failed to detect the river under OLTC V4 while it works well using OLTC V5 (Figs. 7b \& e). Closed-loop trackers have problems to detect the river in such a steep valley, because they depend on the previous measurements. Open-loop trackers should not have this problem, as long as they have an accurate reference elevation. As revealed by the retracked heights of track 67 , the range window was wrongly set at around $3750 \mathrm{~m}$ over the river channel. Because of these settings,S3A recorded only background noises until the update of OLTC V5 that has pre-defined the elevation of VS 35 as $3078 \mathrm{~m}$ (https://www.altimetry-hydro.eu/). Therefore, echoes reflected by the river can be recorded in the waveforms as shown in Fig. 7c. Similarly, track 4 has successfully detected the river although the river width is just $150 \mathrm{~m}$.

(a)

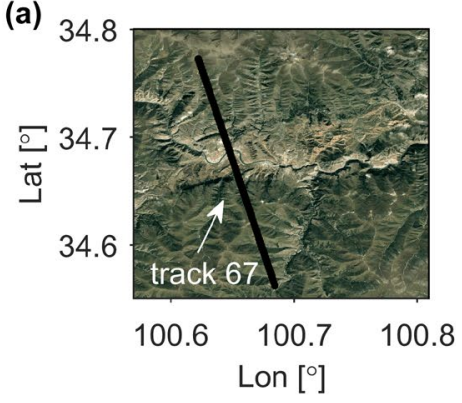

(d)

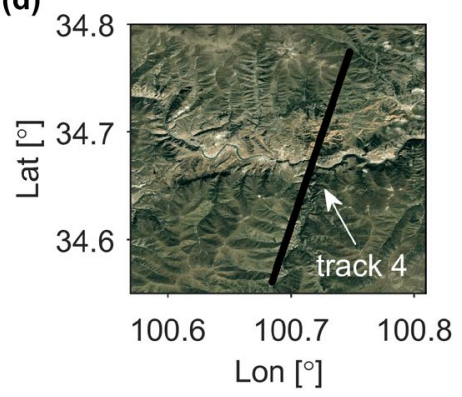

(b)

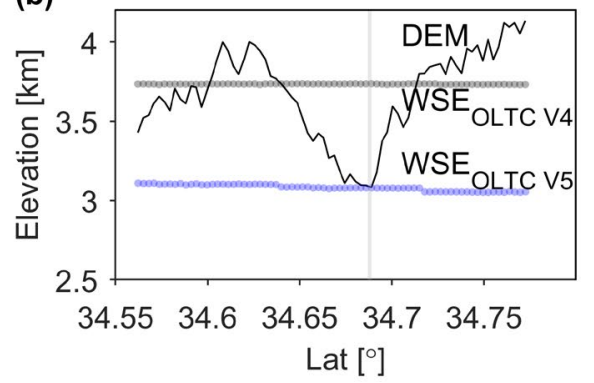

(e)

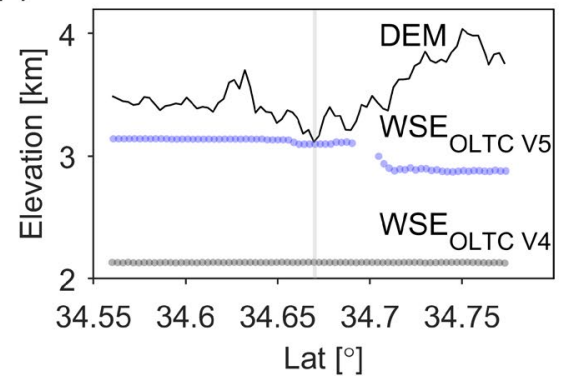

(c)

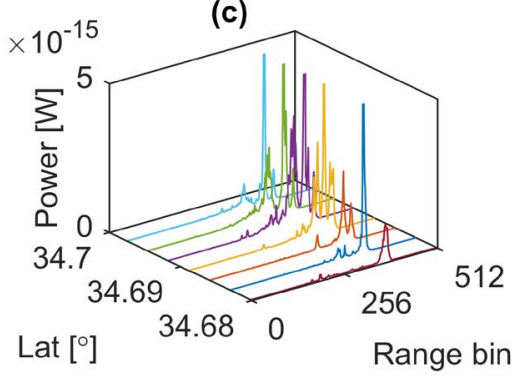

(f)

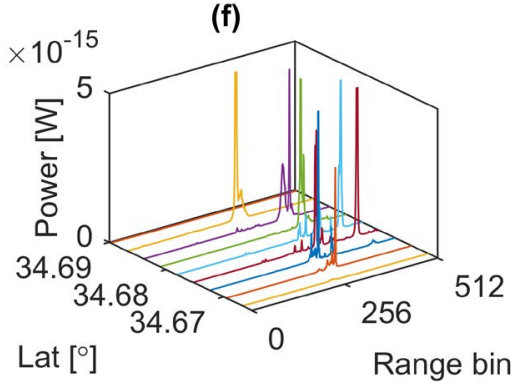

Fig. 7. Comparison of delivered data at VS 36 in open-loop mode under OLTC V4 and OLTC V5. (a) and (d) ground location of track 67 and track 4. (b) and (e) ground elevation from DEM and retracked heights under OLTC V4 and V5. Note that, the river channel is indicated by grey bar. (c) and (f) Several L1b waveforms of track 67 and track 4.

However, OLTC V5 did not solve the problems for all VSs we investigated. At VS 8 and 44 the water surface can still not be detected. At these two locations, no a priori DEM is defined in OLTC V5. The range 
window position is far away from river elevation due to incorrect on-board interpolated DEM. More work is needed to improve the OLTC table. Another shortcoming of current OLTC is that a constant elevation value is given to all locations within and close to a river for each VS. We know that SAR multi-looking process not only uses the look (also called beam) that is taken when the satellite is exactly above a river, but also considers those looks $7.5 \mathrm{~km}$ away from the river. Therefore, the slant range should be within the given value.

\subsection{Performance of retracked L2 WSE}

Due to the invalid waveforms of 11 VSs for the period of July 2016 to March 2019, we only consider the other 39 VSs. Fig. 8 presents the performance of S3A in terms of RMSE of retracked WSE. 19 VSs show good data quality with a RMSE value smaller than $0.6 \mathrm{~m}$, among which 7 VSs show very good agreement with a RMSE $\leq 0.3 \mathrm{~m}$. Another 7 VSs show a moderate RMSE value, ranging from $0.6 \mathrm{~m}$ to $0.9 \mathrm{~m}$. Nevertheless, one third has relatively poor performance regardless of river width. Some of them occur over some tributaries of the Songhua River and the Yangtze River. In the following sections, we will present how different retrackers perform and how the surrounding terrain affects WSE retrieval.

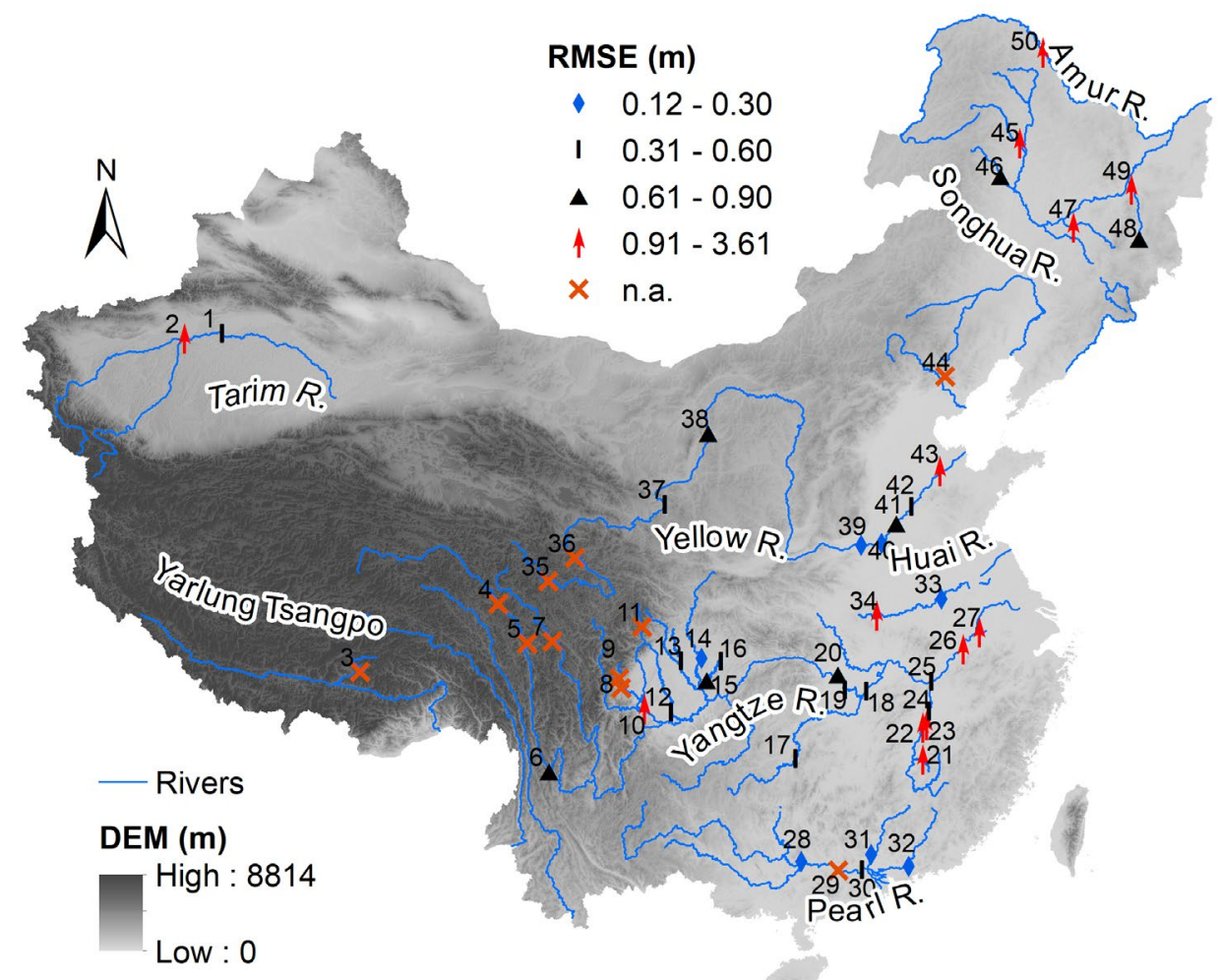

Fig. 8. S3A performance in terms of RMSE of retracked heights against in-situ records. Note that, the best RMSE is used among three (four if available) retrackers. For some VSs, there is no useable data delivered, and therefore no RMSE is available, indicated by n.a. in legend. The categories are based on previously reported accuracy (Table 1 in Villadsen et al., 2016). 


\subsubsection{Comparison of retracking methods}

As shown in Fig. 9, there is no significant difference in terms of average accuracy among SAMOSA+, OCOG, and PPCOG retrackers although they are performing very differently, especially for multi-peak waveforms (e.g. VS 10-17). It should be noted that when calculating RMSE, all observations are used to derive time series. The RMSEs might be smaller if only well-behaved waveforms are used or an ad hoc outlier detection strategy is applied.

Interestingly, all three retrackers consistently perform poorly over the Yangtze River (i.e. VS 18-27) although the river widths are relatively large. An investigation into L1b waveforms reveals that the complexity of waveforms is the reason for the poor performance (See Fig. B1 for an example of multi-peak waveforms). Although the range window was positioned correctly, i.e. signals reflected by the river are contained in the waveforms, the retrackers could not find the range bin which corresponds to the water surface. This is because the multi-peak waveforms reflected by those targets contradict the assumptions of the three retrackers. The SAMOSA+ retracks a modelled waveform which fits a single-peak waveform. Obviously, it incurs inaccuracies when retracking multi-peak waveforms. The OCOG retracker considers the full waveform, but only one peak in the waveform represents the water surface signal. As multiple peaks contribute to the center of gravity (COG), the OCOG retracked elevation is biased. The rationale of PPCOG is that the first peak is the signal from the nadir water surface, but that does not have to be the case if other water surfaces surrounding the river are higher.

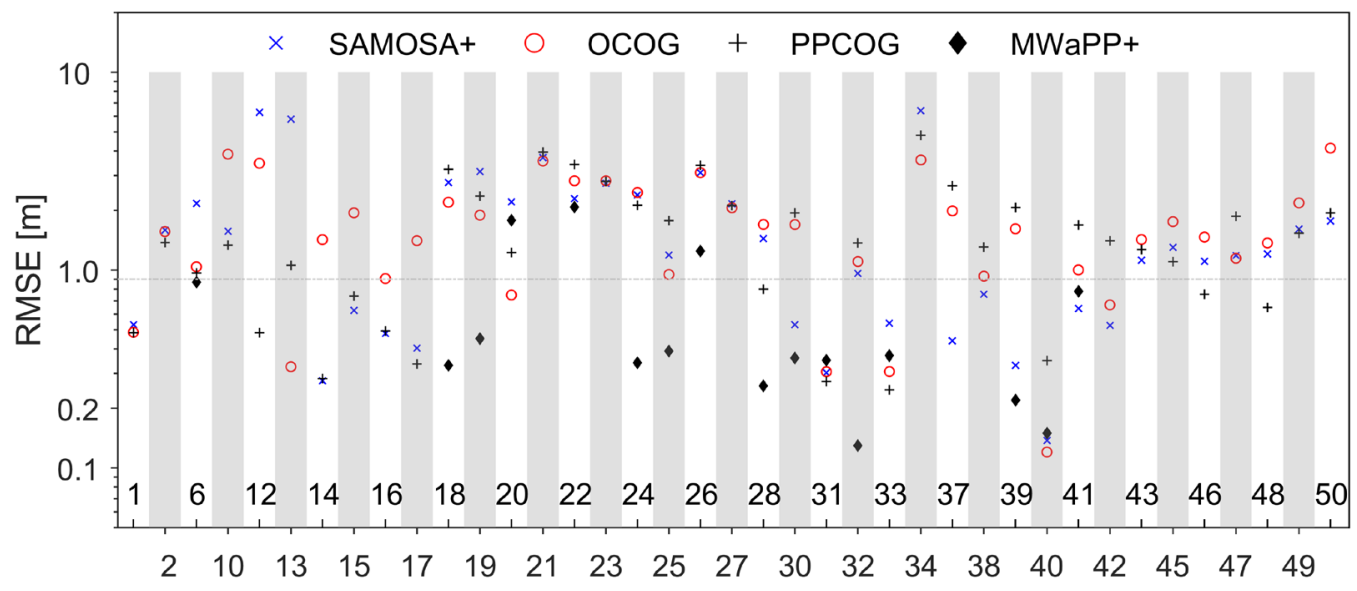

Fig. 9. Comparison of the performance of three (four if applicable) retrackers for $39 \mathrm{VSs}$. Y-axis uses logarithmic scales. X-label indicates the VS ID. Note that, the MWaPP+ is not always applied.

Comparatively, the MWaPP+ works best in most cases (Fig. 9). To demonstrate the differences among retracking methods, five VSs located on the Yangtze River are shown in Fig. 10. Clearly, the MWaPP+ is less sensitive to off-nadir water bodies and other bright targets, while there are large differences between the retracked water levels and the in-situ records for the other three retrackers, especially VS 18, 19, and 25 (Fig. 10). Fig. 10e shows that the three retrackers consistently retrieve WSE of c.a. 9 m. The waveforms are 
probably dominated by other water bodies. In this case, $\mathrm{MWaPP}+$ also has problems to retrack the correct peak reflected by the river. Nevertheless, there is a higher possibility that MWaPP+ retracks the peak reflected by the river, and the retracked WSE outliers are easier to be detected. This approach could potentially be applied to narrow rivers by considering several measurements close to rivers, but the offranging issue needs to be taken into account.

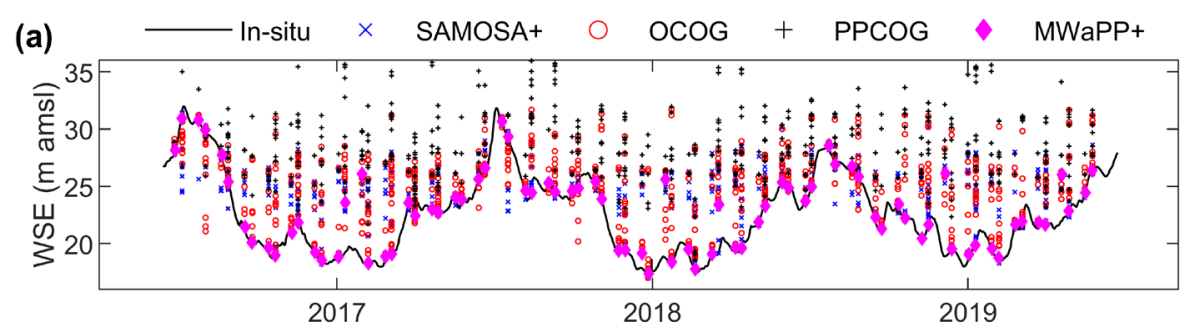

(b)

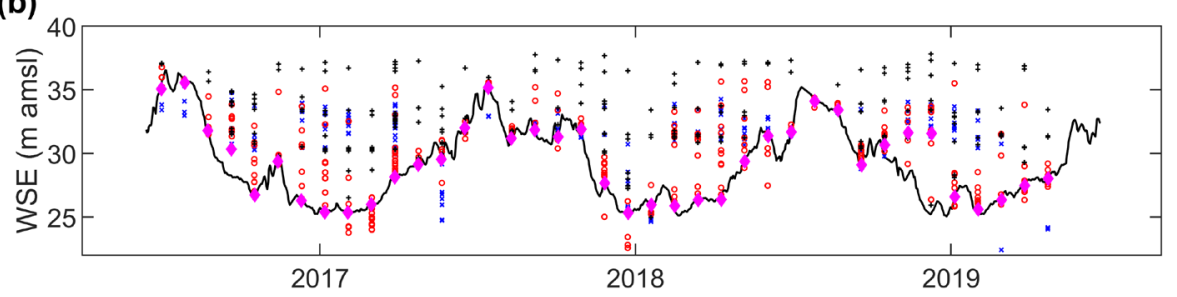

(c)

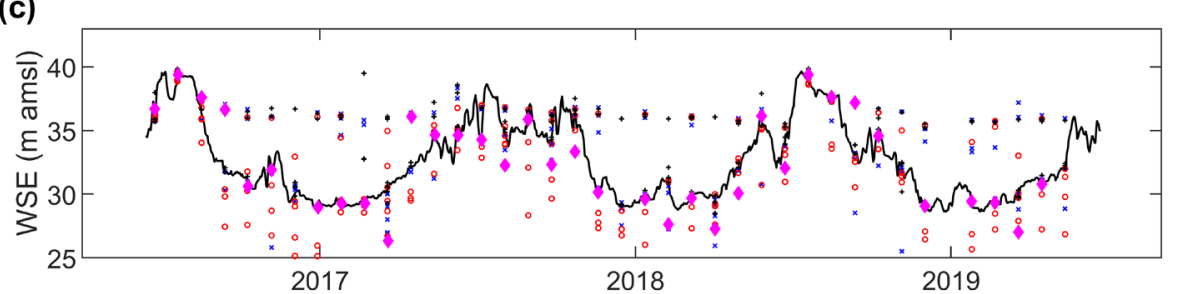

(d)

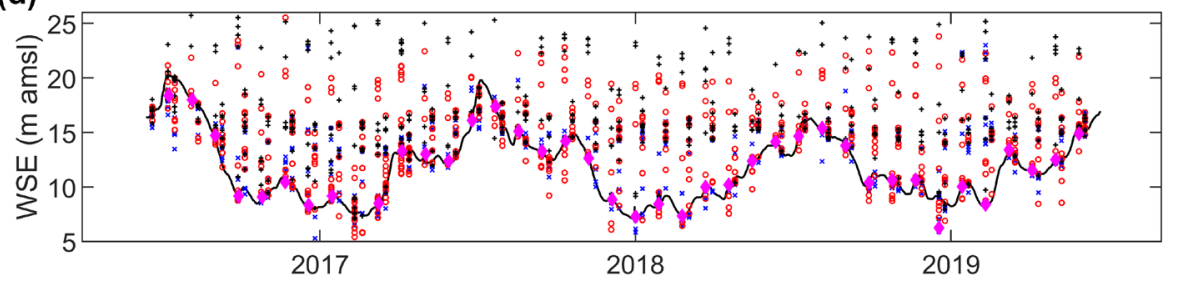

(e)

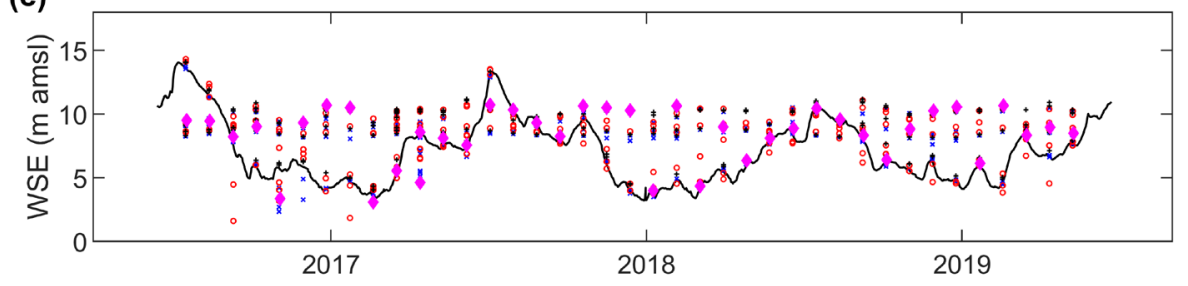

Fig. 10. Comparison of WSE retracted by four methods and gauged water level at five VSs located along

the Yangtze River. (a) - (e) correspond to VS 18 - 20, 25 - 26. Note that, individual retracked WSE is shown for SAMOSA+, OCOG, and PPCOG.

\subsubsection{Influence of river width and surroundings on WSE}

Although it is generally acknowledged that satellite altimetry performs better over large rivers, our results do not show a clear dependence of S3 performance on river width (Fig. 8 and Table A1). Some VSs 
located on a large river, show very noisy waveforms (lower PP values, e.g. VS 10, 20 etc., Fig. 5) and have a poor RMSE (Fig. 9). In contrast, some VSs over medium-sized rivers (e.g. VS 14, 17, 42 etc.) achieve an acceptable performance (RMSE $<0.6 \mathrm{~m}$ ). Some previous studies also reported small RMSE over small rivers. Sulistioadi et al. (2015) reported that the accuracy of Envisat RA-2 over a medium-sized river (width $247 \mathrm{~m}$ ) is around $0.72 \mathrm{~m}$. Kuo and Kao (2011) revealed that the accuracy of river heights retrieved from Jason-2 over a small river $(<200 \mathrm{~m}$ in dry season) is $0.31 \mathrm{~m}$.

As shown previously, the quality of retracked WSE over larger rivers, e.g. the Yangtze river, is not necessarily better than medium rivers. Given that the footprint of satellite radar altimeters is relatively large (15 km across-track and $300 \mathrm{~m}$ along-track for S3), the ability to detect a river is dependent on river width and the orientation with respect to the satellite ground track (Jiang et al., 2017b); and to a larger extent, data quality is determined by the environmental factors, such as the surrounding topographic relief, land cover type surrounding the rivers, as well as the instrument design and configuration (Biancamaria et al., 2018; Maillard et al., 2015). The environmental factors can seriously contaminate waveforms, making it difficult to retrieve correct WSE. For many of the investigated VSs with a large RMSE, the echoes are not singlepeak waveforms. These contaminated waveforms could be attributed to small lakes, sand banks, building roofs, ships, etc. (Biancamaria et al., 2017; Gómez-Enri et al., 2016).

Moreover, environmental factors can also cause the "snagging" effect, where the retrievals do not represent the correct WSE of rivers. The noisy waveforms at VS e.g. 22, 24, are caused by snagging, e.g. altimeter locking on off-nadir targets. As shown in Fig. 11, there are many small lakes close to the river, and their elevations are higher than river WSE. Therefore, the waveform is dominated by echoes reflected by small lakes which usually have strong reflectance due to a calm surface. This explains the wrong retracked WSE which shows no obvious seasonality as shown in Fig. 11.

This effect could be eliminated through a careful analysis of waveforms and discarding invalid measurements. For example, a priori information, such as historical river stage records, could also help to filter outliers out although a lot of measurements will be lost. However, this kind of approaches does not solve the problem fundamentally. By discarding some contaminated waveforms, those methods indeed improve the overall accuracy, but reduce the number of measurements in a time series (Armitage and Davidson, 2014). Alternatively, a dedicated sub-waveform selection strategy could be used to extract the target sub-waveform, e.g. last peak in the cases shown in Fig. 11. Another approach could be applied is the fully focused SAR, which can obtain an along-track resolution of $0.5 \mathrm{~m}$ (Egido and Smith, 2016). This smaller footprint could differentiate different targets along-track but still be subject to across-track heterogeneous surfaces. Technically, this issue can be potentially solved by across-track interferometry if future missions can operate in SARIn mode. This has been demonstrated for the CryoSat-2 SARIn over coastal regions and sea ice (Abulaitijiang et al., 2015; Armitage and Davidson, 2014). 

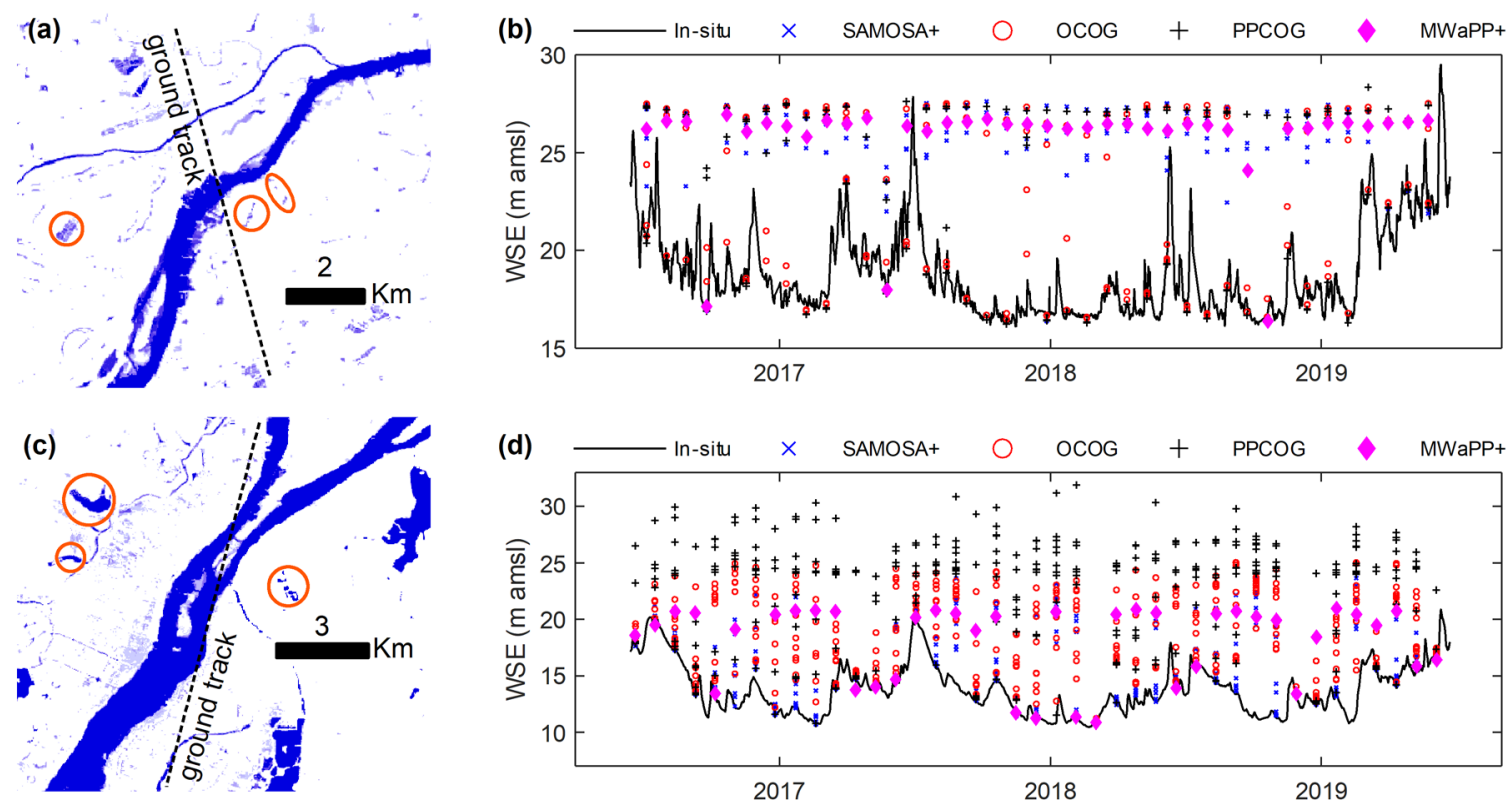

371

Fig. 11. Exemplary snagging effect on VS 22 (a and b) and 24 (c and d). (a) and (b) show the water bodies using Global Surface Water Explorer (https://global-surface-water.appspot.com/map). Some small lakes close by are highlighted. (c) and (d) show retracked WSE against in-situ records.

\section{Summary and conclusions}

Satellite radar altimetry is now widely used by hydrologists for the purposes of surface water monitoring and hydrologic/hydrodynamic model calibrations, especially in poorly ungauged regions and transboundary basins, where in-situ gauge records may be classified. However, it is well known that altimeters have difficulties to track rivers in mountain areas. To overcome this issue, an open-loop tracking mode is available on Sentinel-3A (S3A) where the altimeter range window is positioned using a-priori information of the surface height. In this way, tracking of rivers in rugged topography can be guaranteed in principle.

In this study, we have evaluated the performance of S3A across diverse rivers (50 virtual stations (VS)) in China in terms of Level 1 data and Level 2 retracked water surface elevation (WSE). In the following we summarize this study to answer the questions posed in the introduction, i.e. Can S3A deliver reliable measurements of WSE for mountain rivers? What is the accuracy of S3A derived WSE? Can S3A observe medium rivers (of width around or smaller than $300 \mathrm{~m}$ )?

Whether a river can be measured depends on the altimeter's tracking function. Valid signals are recorded by the altimeter only if the elevation of a river is within its range window. The evaluation is based on several metrics derived from L1 data. We found that S3A did not deliver useful Level 1 data for 11 VSs prior to $1^{\text {st }}$ March 2019, which are mainly located in mountain areas. Investigation shows that the main problem can be attributed to the incorrect on-board pseudo-DEM (OLTC V4), which leads to a wrongly positioned range 
window. Therefore, Level 1 data do not enclose useful echoes reflected by rivers. Further investigation reveals that valid data has been delivered for most VSs after the update of on-board DEM table, i.e. OLTC V5. Therefore, S3A is capable of delivering WSE for mountain rivers if the corresponding VSs are defined in OLTC V5 and appropriate target elevation is available on-board.

We implemented different retracking methods to retrieve Level 2 WSE for 39 VSs. Specifically, 19 (49\%) VSs show small RMSE values ranging from 0.12 to $0.6 \mathrm{~m}$. Seven (18\%) VSs achieve moderate RMSE values (0.6 - $0.9 \mathrm{~m})$. The remaining 13 (33\%) VSs show relatively larger RMSE values, ranging from $0.9 \mathrm{~m}$ to a few meters. The comparison reveals that SAMOSA+, OCOG, and PPCOG perform poorly for some of the VSs, especially those located along the Yangtze and the Pearl Rivers. Analysis of the waveforms reveal that the main reason is due to multi-peak waveforms. This makes it difficult for the retracker to find the correct bin, which corresponds to echoes from river itself. By taking advantage of persistent peak in adjacent waveforms, as implemented in MWaPP+ retracker, we are able to improve the retracking of the correct bin in most cases. This method shows significant advantages over the other three methods for river WSE retrieval.

In addition, our results show no clear dependence of measurement performance on the river width. S3A can deliver measurements for medium rivers as good as large ones. It should be noted that the surrounding terrain is the main factor determining whether the WSE can be successfully retrieved. Snagging is a major issue for the VSs in the Yangtze river basin due to widely distributed small lakes in the vicinity of the river. In this regard, we suggest using MWaPP+ or similar methods for retracking. However, there is no one-sizefits-all retracking approach for different rivers considering the width, flow direction relative to the orbit orientation, and surrounding terrain.

\section{Acknowledgements}

This work is partly funded by the Ministry of Foreign Affairs of Denmark and administered by Danida Fellowship Centre (EOForChina project, File number: 18-M01-DTU). We would like to acknowledge Giovanni Sabatino for his assistance on the GPOD/SARvatore service. We also thank Sophie Le Gac for her explanation on OLTC. We are grateful to three anonymous reviewers for their comments and suggestions.

\section{Appendix A}

Table A1. Characteristics of each virtual station and the performance of different retracking methods (rows greyed out indicate that L1 data are invalid for the period prior to $1^{\text {st }}$ March 2019).

\begin{tabular}{|c|c|c|c|c|c|c|c|c|}
\hline $\begin{array}{l}\text { VS } \\
\text { ID }\end{array}$ & Lon $\left(^{\circ}\right)$ & Lat $\left({ }^{\circ}\right)$ & $\begin{array}{c}\text { Altitude } \\
(\mathrm{m} \text { amsl) }\end{array}$ & $\begin{array}{c}\text { River } \\
\text { width } \\
(\mathrm{m})\end{array}$ & $\begin{array}{c}\text { SAMOSA+ } \\
\text { RMSE (m) }\end{array}$ & $\begin{array}{c}\text { OCOG } \\
\text { RMSE (m) }\end{array}$ & $\begin{array}{c}\text { PPCOG } \\
\text { RMSE (m) }\end{array}$ & $\begin{array}{c}\text { MWaPP+ } \\
\text { RMSE (m) }\end{array}$ \\
\hline $\mathbf{1}$ & 82.747 & 41.029 & 966 & $100-600$ & 0.53 & $\mathbf{0 . 4 8}$ & $\mathbf{0 . 4 8}$ & - \\
\hline $\mathbf{2}$ & 81.05 & 40.53 & 1017 & $40-520$ & 1.59 & 1.56 & $\mathbf{1 . 3 8}$ & - \\
\hline $\mathbf{3}$ & 91.726 & 29.868 & 3813 & $100-1300$ & - & - & - & - \\
\hline $\mathbf{4}$ & 97.384 & 32.837 & 3480 & 150 & - & - & - & - \\
\hline
\end{tabular}




\begin{tabular}{|c|c|c|c|c|c|c|c|c|}
\hline 5 & 98.759 & 31.489 & 3001 & 170 & - & - & - & - \\
\hline 6 & 100 & 26.885 & 1810 & 500 & 2.17 & 1.04 & 0.97 & 0.87 \\
\hline 7 & 99.845 & 31.624 & 3370 & 80 & - & - & - & - \\
\hline 8 & 102.811 & 30.368 & 990 & 60 & - & - & - & - \\
\hline 9 & 102.905 & 30.018 & 611 & 65 & - & - & - & - \\
\hline 10 & 103.898 & 29.271 & 325 & 470 & 1.57 & 3.85 & 1.34 & - \\
\hline 11 & 103.761 & 32.246 & 2401 & 30 & - & - & - & - \\
\hline 12 & 105.006 & 29.186 & 266 & 220 & 6.28 & 3.47 & 0.48 & - \\
\hline 13 & 105.431 & 31.034 & 352 & 160 & 5.81 & 0.32 & 1.06 & - \\
\hline 14 & 106.342 & 31.123 & 294 & 270 & 0.28 & 1.43 & 0.28 & - \\
\hline 15 & 106.566 & 30.311 & 203 & 330 & 0.63 & 1.95 & 0.74 & - \\
\hline 16 & 107.155 & 31.001 & 240 & 390 & 0.48 & 0.91 & 0.49 & - \\
\hline 17 & 110.163 & 27.359 & 148 & 320 & 0.40 & 1.41 & 0.34 & - \\
\hline 18 & 113.316 & 29.648 & 19 & 2000 & 2.78 & 2.20 & 3.23 & 0.33 \\
\hline 19 & 112.42 & 29.749 & 25 & 760 & 3.15 & 1.90 & 2.37 & 0.45 \\
\hline 20 & 112.179 & 30.308 & 33 & 1400 & 2.21 & 0.75 & 1.23 & 1.79 \\
\hline 21 & 115.418 & 26.967 & 86 & 70 & 3.71 & 3.56 & 3.94 & - \\
\hline 22 & 115.571 & 28.121 & 17 & 600 & 2.30 & 2.83 & 3.42 & 2.08 \\
\hline 23 & 115.741 & 28.19 & 15 & 420 & 2.75 & 2.83 & 2.80 & - \\
\hline 24 & 115.876 & 28.694 & 12 & 960 & 2.40 & 2.47 & 2.13 & 0.34 \\
\hline 25 & 116.121 & 29.763 & 10 & 2000 & 1.19 & 0.95 & 1.78 & 0.39 \\
\hline 26 & 117.656 & 30.781 & 1 & 2000 & 3.11 & 3.11 & 3.38 & 1.25 \\
\hline 27 & 118.452 & 31.316 & 5 & 180 & 2.17 & 2.07 & 2.12 & - \\
\hline 28 & 110.203 & 23.565 & 20 & 580 & 1.44 & 1.70 & 0.80 & 0.26 \\
\hline 29 & 111.643 & 23.163 & 0 & 750 & - & - & - & - \\
\hline 30 & 112.568 & 23.126 & 0 & 410 & 0.53 & 1.70 & 1.95 & - \\
\hline 31 & 112.979 & 23.673 & 5 & 1000 & 0.30 & 0.31 & 0.27 & 0.35 \\
\hline 32 & 114.433 & 23.111 & 7 & 600 & 0.96 & 1.11 & 1.37 & 0.13 \\
\hline 33 & 116.976 & 32.679 & 17 & 550 & 0.54 & 0.31 & 0.25 & 0.37 \\
\hline 34 & 114.074 & 32.324 & 69 & 300 & 6.39 & 3.60 & 4.80 & - \\
\hline 35 & 99.529 & 33.787 & 3974 & 750 & - & - & - & - \\
\hline 36 & 100.677 & 34.677 & 3076 & 150 & - & - & - & - \\
\hline 37 & 104.722 & 36.681 & 1382 & 210 & 0.44 & 1.99 & 2.67 & - \\
\hline 38 & 106.802 & 39.222 & 1088 & 480 & 0.75 & 0.93 & 1.31 & - \\
\hline 39 & 113.664 & 34.91 & 91 & 700 & 0.33 & 1.62 & 2.07 & 0.22 \\
\hline 40 & 114.601 & 34.913 & 72 & 680 & 0.14 & 0.12 & 0.35 & 0.15 \\
\hline 41 & 115.349 & 35.559 & 52 & 500 & 0.64 & 1.00 & 1.69 & 0.78 \\
\hline 42 & 116.114 & 36.132 & 38 & 300 & 0.53 & 0.67 & 1.40 & - \\
\hline 43 & 117.634 & 37.26 & 13 & 300 & 1.12 & 1.43 & 1.27 & - \\
\hline 44 & 118.485 & 40.614 & 297 & 160 & - & - & - & - \\
\hline 45 & 124.257 & 48.536 & 203 & 310 & 1.31 & 1.76 & 1.10 & - \\
\hline 46 & 122.883 & 47.48 & 212 & 220 & 1.11 & 1.47 & 0.75 & - \\
\hline 47 & 126.159 & 45.132 & 135 & 160 & 1.19 & 1.15 & 1.87 & - \\
\hline 48 & 129.326 & 44.172 & 255 & 190 & 1.21 & 1.37 & 0.65 & - \\
\hline 49 & 129.567 & 46.003 & 110 & 290 & 1.62 & 2.19 & 1.54 & - \\
\hline 50 & 126.582 & 51.656 & 168 & 180 & 1.77 & 4.14 & 1.95 & - \\
\hline
\end{tabular}



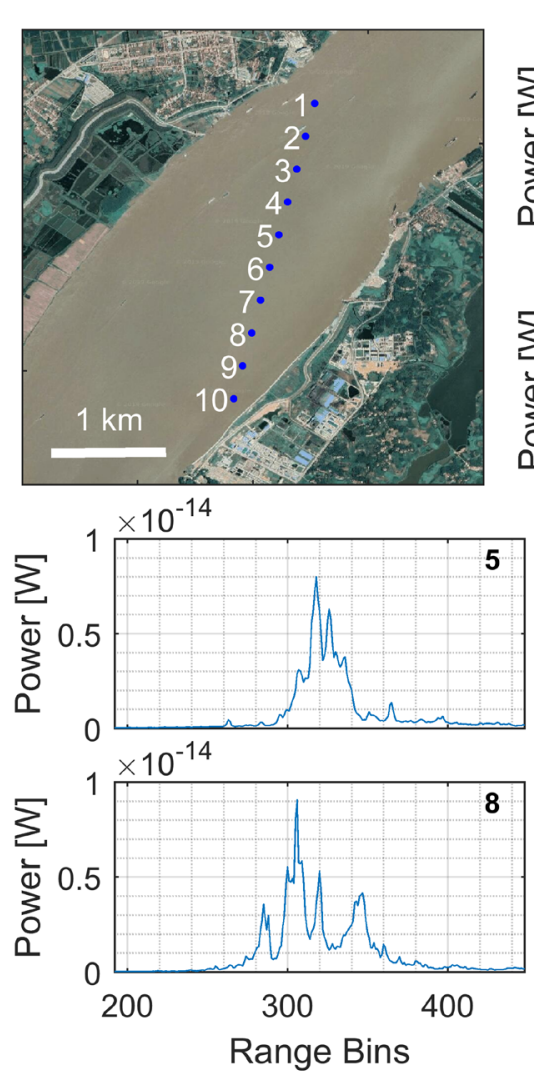
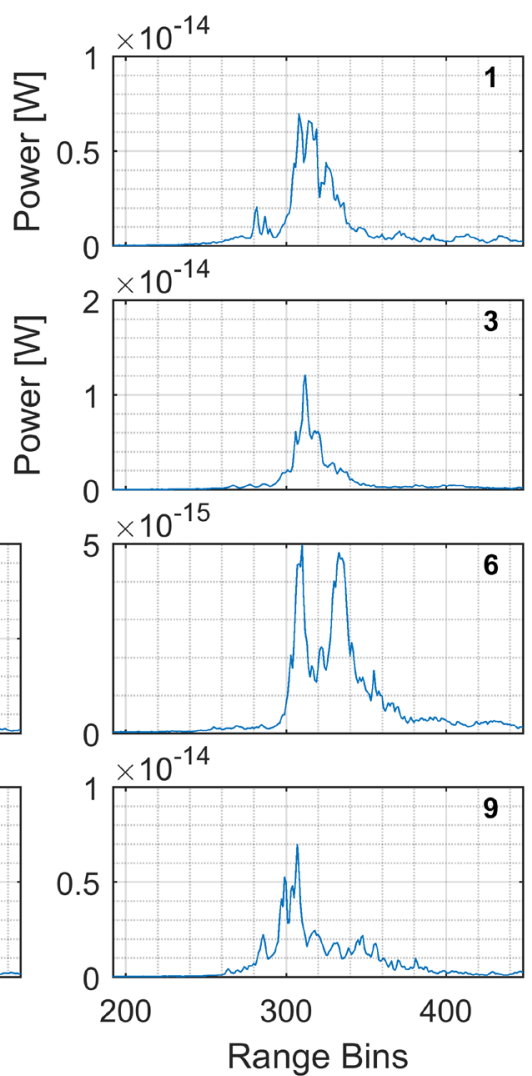
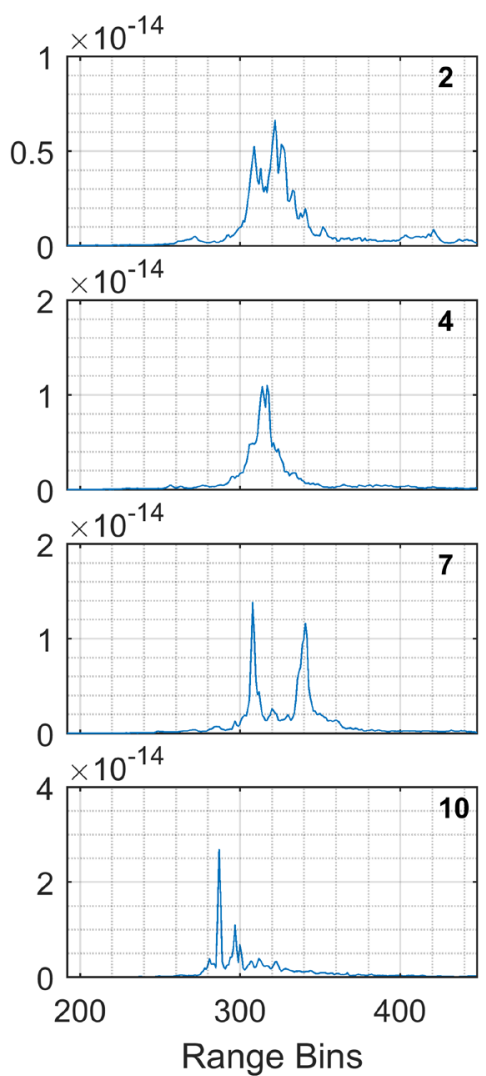

Fig. B1. An example of waveforms with multiple peaks at VS 18, downstream of the Yangtze River.

Only part of the full waveform is shown in each plot for clarity.

\section{References}

Abulaitijiang, A., Andersen, O.B., Stenseng, L., 2015. Coastal sea level from inland CryoSat-2 interferometric SAR altimetry. Geophys. Res. Lett. 42, 1841-1847. https://doi.org/10.1002/2015GL063131

Armitage, T.W.K., Davidson, M.W.J., 2014. Using the interferometric capabilities of the ESA CryoSat-2 mission to improve the accuracy of sea ice freeboard retrievals. IEEE Trans. Geosci. Remote Sens. 52, 529-536. https://doi.org/10.1109/TGRS.2013.2242082

Arnell, N.W., Gosling, S.N., 2013. The impacts of climate change on river flow regimes at the global scale. J. Hydrol. 486, 351-364. https://doi.org/10.1016/j.jhydrol.2013.02.010

Berry, P.A.M., Garlick, J.D., Freeman, J.A., Mathers, E.L., 2005. Global inland water monitoring from multi-mission altimetry. Geophys. Res. Lett. 32, 1-4. https://doi.org/10.1029/2005GL022814

Biancamaria, S., Frappart, F., Leleu, A.-S., Marieu, V., Blumstein, D., Desjonquères, J.-D., Boy, F., Sottolichio, A., Valle-Levinson, A., 2017. Satellite radar altimetry water elevations performance over a 200m wide river: Evaluation over the Garonne River. Adv. Sp. Res. 59, 128-146. https://doi.org/10.1016/j.asr.2016.10.008 
Biancamaria, S., Schaedele, T., Blumstein, D., Frappart, F., Boy, F., Desjonquères, J.D., Pottier, C., Blarel, F., Niño, F., 2018. Validation of Jason-3 tracking modes over French rivers. Remote Sens. Environ. 209, 77-89. https://doi.org/10.1016/j.rse.2018.02.037

Blumstein, D., Lasson, L., Gac, S. Le, Biancamaria, S., Calmant, S., Crétaux, J.F., Blarel, F., Frappart, F., Papa, F., Kouraev, A. V., Paris, A., Zawadski, L., Boy, F., Picot, N., Femenias, P., 2019. Major upgrade of OLTC on Sentinel-3A and 3B in 2018: benefits for inland waters users, in: Living Planet Symposium.

Chelton, D.B., Ries, J.C., Haines, B.J., Fu, L.L., Callahan, P.S., 2001. Chapter 1 Satellite Altimetry, in: International Geophysics. https://doi.org/10.1016/S0074-6142(01)80146-7

Dehecq, A., Gourmelen, N., Shepherd, A., Cullen, R., Trouvé, E., 2013. Evaluation of CryoSat-2 for height retrieval over the Himalayan range, in: CryoSat-2 Third User Workshop, March 2013. Dresden, Germany.

Desjonquères, J.D., Carayon, G., Steunou, N., Lambin, J., 2010. Poseidon-3 Radar Altimeter: New Modes and In-Flight Performances. Mar. Geod. 33, 53-79. https://doi.org/10.1080/01490419.2010.488970

Dinardo, S., Benveniste, J., 2013. Guidelines for the SAR (Delay-Doppler) L1b Processing.

Dinardo, S., Fenoglio-Marc, L., Buchhaupt, C., Becker, M., Scharroo, R., Joana Fernandes, M., Benveniste, J., 2018. Coastal SAR and PLRM altimetry in German Bight and West Baltic Sea. Adv. Sp. Res. 62, 1371-1404. https://doi.org/10.1016/j.asr.2017.12.018

Dinardo, S., Lucas, B., Benveniste, J., 2014. SAR altimetry processing on demand service for CryoSat-2 at ESA G-POD, in: Proceeding of the 2014 Conference on Big Data from Space (BiDS'14). pp. 299302. https://doi.org/10.2788/1823

Domeneghetti, A., Tarpanelli, A., Brocca, L., Barbetta, S., Moramarco, T., Castellarin, A., Brath, A., 2014. The use of remote sensing-derived water surface data for hydraulic model calibration. Remote Sens. Environ. 149, 130-141. https://doi.org/10.1016/j.rse.2014.04.007

Egido, A., Smith, W.H.F., 2016. Fully Focused SAR Altimetry: Theory and Applications. IEEE Trans. Geosci. Remote Sens. 55, 1-15. https://doi.org/10.1109/TGRS.2016.2607122

Gómez-Enri, J., Scozzari, A., Soldovieri, F., Coca, J., Vignudelli, S., 2016. Detection and characterization of ship targets using CryoSat-2 altimeter waveforms. Remote Sens. 8, 1-13. https://doi.org/10.3390/rs8030193

Huang, Q., Li, X., Han, P., Long, D., Zhao, F., Hou, A., 2019. Validation and application of water levels derived from Sentinel-3A for the Brahmaputra River. Sci. China Technol. Sci. 6-8. 
https://doi.org/10.1007/s11431-019-9535-3

472

473

474

475

476

477

478

479

480

481

482

483

484

485

486

487

488

489

490

491

492

493

494

495

496

497

498

499

500

Idžanović, M., Ophaug, V., Andersen, O.B., 2018. Coastal sea level from CryoSat-2 SARIn altimetry in Norway. Adv. Sp. Res. 62, 1344-1357. https://doi.org/10.1016/j.asr.2017.07.043

Immerzeel, W.W., Van Beek, L.P.H., Bierkens, M.F.P., 2010. Climate change will affect the Asian water towers. Science (80-. ). 328, 1382-1385.

Jain, M., Andersen, O.B., Dall, J., Stenseng, L., 2015. Sea surface height determination in the Arctic using Cryosat-2 SAR data from primary peak empirical retrackers. Adv. Sp. Res. 55, 40-50. https://doi.org/10.1016/j.asr.2014.09.006

Jiang, L., Andersen, O.B., Nielsen, K., Zhang, G., Bauer-Gottwein, P., 2019a. Influence of local geoid variation on water surface elevation estimates derived from multi-mission altimetry for Lake Namco. Remote Sens. Environ. 221, 65-79. https://doi.org/10.1016/j.rse.2018.11.004

Jiang, L., Madsen, H., Bauer-Gottwein, P., 2019b. Simultaneous calibration of multiple hydrodynamic model parameters using satellite altimetry observations of water surface elevation in the Songhua River. Remote Sens. Environ. 225, 229-247. https://doi.org/10.1016/j.rse.2019.03.014

Jiang, L., Nielsen, K., Andersen, O.B., Bauer-Gottwein, P., 2017a. CryoSat-2 radar altimetry for monitoring China’s freshwater resources. Remote Sens. Environ. 200, 125-139. https://doi.org/10.1016/j.rse.2017.08.015

Jiang, L., Schneider, R., Andersen, O.B., Bauer-Gottwein, P., 2017b. CryoSat-2 Altimetry Applications over Rivers and Lakes. Water 9, 211. https://doi.org/10.3390/w9030211

Kittel, C.M.M., Nielsen, K., Tøttrup, C., Bauer-Gottwein, P., 2018. Informing a hydrological model of the Ogooué with multi-mission remote sensing data. Hydrol. Earth Syst. Sci. 22, 1453-1472. https://doi.org/10.5194/hess-22-1453-2018

Kleinherenbrink, M., Lindenbergh, R.C., Ditmar, P.G., 2015. Monitoring of lake level changes on the Tibetan Plateau and Tian Shan by retracking Cryosat SARIn waveforms. J. Hydrol. 521, 119-131. https://doi.org/10.1016/j.jhydrol.2014.11.063

Krasovskaia, I., Gottschalk, L., 2002. River flow regimes in a changing climate. Hydrol. Sci. J. 47, 597609. https://doi.org/10.1080/02626660209492962

Kuo, C.-Y., Kao, H.-C., 2011. Retracked Jason-2 Altimetry over Small Water Bodies: Case Study of Bajhang River, Taiwan. Mar. Geod. 34, 382-392. https://doi.org/10.1080/01490419.2011.584830

Leon, J.G., Calmant, S., Seyler, F., Bonnet, M.-P., Cauhopé, M., Frappart, F., Filizola, N., Fraizy, P., 2006. 
Rating curves and estimation of average water depth at the upper Negro River based on satellite altimeter data and modeled discharges. J. Hydrol. 328, 481-496. https://doi.org/10.1016/j.jhydrol.2005.12.006

Liu, G., Schwartz, F.W., Tseng, K.-H., Shum, C.K., 2015. Discharge and water-depth estimates for ungauged rivers: Combining hydrologic, hydraulic, and inverse modeling with stage and water-area measurements from satellites. Water Resour. Res. 51, 6017-6035. https://doi.org/10.1002/2015WR016971

Maillard, P., Bercher, N., Calmant, S., 2015. New processing approaches on the retrieval of water levels in Envisat and SARAL radar altimetry over rivers: A case study of the São Francisco River, Brazil. Remote Sens. Environ. 156, 226-241. https://doi.org/10.1016/j.rse.2014.09.027

Michailovsky, C.I., McEnnis, S., Berry, P.A.M., Smith, R., Bauer-Gottwein, P., 2012. River monitoring from satellite radar altimetry in the Zambezi River basin. Hydrol. Earth Syst. Sci. 16, 2181-2192. https://doi.org/10.5194/hess-16-2181-2012

Michailovsky, C.I., Milzow, C., Bauer-Gottwein, P., 2013. Assimilation of radar altimetry to a routing model of the Brahmaputra River. Water Resour. Res. 49, 4807-4816. https://doi.org/10.1002/wrcr.20345

Nielsen, K., Stenseng, L., Andersen, O.B., Knudsen, P., 2017. The Performance and Potentials of the CryoSat-2 SAR and SARIn Modes for Lake Level Estimation. Water 9, 374. https://doi.org/10.3390/w9060374

Nielsen, K., Stenseng, L., Andersen, O.B., Villadsen, H., Knudsen, P., 2015. Validation of CryoSat-2 SAR mode based lake levels. Remote Sens. Environ. 171, 162-170. https://doi.org/10.1016/j.rse.2015.10.023

Normandin, C., Frappart, F., Diepkilé, A.T., Marieu, V., Mougin, E., Blarel, F., Lubac, B., Braquet, N., Ba, A., 2018. Evolution of the Performances of Radar Altimetry Missions from ERS-2 to Sentinel-3A over the Inner Niger Delta. Remote Sens. 10, 833. https://doi.org/10.3390/rs10060833

Pekel, J.-F., Cottam, A., Gorelick, N., Belward, A.S., 2016. High-resolution mapping of global surface water and its long-term changes. Nature 1-19. https://doi.org/10.1038/nature20584

Rosmorduc, V., Benveniste, J., Lauret, O., Maheu, C., Milagro, M., Picot, N., 2011. Radar altimetry tutorial, Http://www.altimetry.info.

Sauer, V.B., Turnipseed, D.P., 2010. Stage measurement at gaging stations. US Department of the Interior, US Geological Survey. 
Schneider, R., Ridler, M.E., Godiksen, P.N., Madsen, H., Bauer-Gottwein, P., 2018a. A data assimilation system combining CryoSat-2 data and hydrodynamic river models. J. Hydrol. 557, 197-210. https://doi.org/10.1016/j.jhydrol.2017.11.052

Schneider, R., Tarpanelli, A., Nielsen, K., Madsen, H., Bauer-Gottwein, P., 2018b. Evaluation of multimode CryoSat-2 altimetry data over the Po River against in situ data and a hydrodynamic model. Adv. Water Resour. 112, 17-26. https://doi.org/10.1016/j.advwatres.2017.11.027

Sichangi, A.W., Wang, L., Yang, K., Chen, D., Wang, Z., Li, X., Zhou, J., Liu, W., Kuria, D., 2016. Estimating continental river basin discharges using multiple remote sensing data sets. Remote Sens. Environ. 179, 36-53. https://doi.org/10.1016/j.rse.2016.03.019

Sulistioadi, Y.B., Tseng, K.-H., Shum, C.K., Hidayat, H., Sumaryono, M., Suhardiman, A., Setiawan, F., Sunarso, S., 2015. Satellite radar altimetry for monitoring small rivers and lakes in Indonesia. Hydrol. Earth Syst. Sci. 19, 341-359. https://doi.org/10.5194/hess-19-341-2015

Tarpanelli, A., Barbetta, S., Brocca, L., Moramarco, T., 2013. River discharge estimation by using altimetry data and simplified flood routing modeling. Remote Sens. 5, 4145-4162. https://doi.org/10.3390/rs5094145

Villadsen, H., Deng, X., Andersen, O.B., Stenseng, L., Nielsen, K., Knudsen, P., 2016. Improved inland water levels from SAR altimetry using novel empirical and physical retrackers. J. Hydrol. 537, 234 247. https://doi.org/10.1016/j.jhydrol.2016.03.051

Wingham, D.J., Francis, C.R., Baker, S., Bouzinac, C., Brockley, D., Cullen, R., de Chateau-Thierry, P., Laxon, S.W., Mallow, U., Mavrocordatos, C., Phalippou, L., Ratier, G., Rey, L., Rostan, F., Viau, P., Wallis, D.W., 2006. CryoSat: A mission to determine the fluctuations in Earth's land and marine ice fields. Adv. Sp. Res. 37, 841-871. https://doi.org/10.1016/j.asr.2005.07.027 